\title{
Verificação de um modelo computacional para a flambagem elástica de placas ortotrópicas
}

Verification of a computational model for the elastic buckling of orthotropic plates

\author{
J. P. S. Lima ${ }^{1}$; A. P. Ramos ${ }^{1}$; F. S. Carvalho ${ }^{2}$; L. A. O. Rocha ${ }^{3}$; E. D. dos Santos ${ }^{1}$; \\ M. V. Real ${ }^{1}$; L. A. Isoldi ${ }^{1}$ \\ IPrograma de Pós-Graduação em Engenharia Oceânica, Universidade Federal do Rio Grande, 96.201-900, Rio \\ Grande-Rio Grande do Sul, Brasil \\ ${ }^{2}$ Departamento de Engenharia Civil, Universidade Federal Triângulo Mineiro, 38.064-200, Uberaba-Minas Gerais, \\ Brasil
}

${ }_{3}^{3}$ Programa de Pós-Graduação em Engenharia Mecânica, Universidade Federal do Rio Grande do Sul, 90.050-170, Porto Alegre-Rio Grande do Sul, Brasil

*joaop_1005@hotmail.com

(Recebido em 10 de maio de 2017; aceito em 22 de maio de 2017)

\begin{abstract}
Placas ortotrópicas possuem vigas dispostas longitudinalmente e transversalmente, chamadas de enrijecedores, com o objetivo de aumentar a resistência mecânica. Porém, a presença de enrijecedores acarreta em dificuldades na análise da tensão crítica de flambagem, uma vez que se tem uma gama maior de variáveis a serem analisadas, além de se tornarem os modos de flambagem mais complexos. Tendo essa dificuldade na solução analítica para placas enrijecidas, métodos numéricos e experimentais se tornam essenciais. Sendo assim, empregou-se o Método dos Elementos Finitos, utilizando o software ANSYS para a análise da tensão crítica de flambagem de placas com enrijecedores. Os resultados obtidos foram comparados com as soluções analíticas e aproximadas disponíveis na literatura, mostrando uma fidelidade entre os resultados numéricos do modelo proposto e as soluções analíticas, verificando assim o modelo computacional proposto.

Palavras-chave: Flambagem, Placa, Enrijecedores
\end{abstract}

Orthotropic plates have beams arranged longitudinally and transversely, called stiffeners, in order to increase mechanical strength. However, the stiffeners cause difficulties in the analysis of the critical buckling stress, since there is a larger range of variables to be analyzed, in addition to turning more complex the buckling modes. Through this difficulty in the analytical solution for stiffened plates, numerical and experimental methods become essential. Thus, the Finite Element Method was used, using the software ANSYS to analyze the critical buckling stress of plates with stiffeners. The obtained results were compared with the analytical and approximate solutions available in the literature, showing a fidelity between the numerical results of the proposed model and the analytical solutions, thus verifying the proposed computational model.

Keywords: Buckling, Plates, Stiffeners

\section{INTRODUÇÃO}

Placas e painéis enrijecidos são aplicados por muitos anos em vários setores da engenharia estrutural. Na indústria aeroespacial, eles são usados na fuselagem e na produção de aeronaves, enquanto que na indústria naval, são utilizados na construção de cascos de navios. Placas enrijecidas são também utilizadas na construção de pontes, edifícios, estruturas off-shore e recentemente aplicados em instalações de processamento petroquímico. O sistema estrutural é composto pela placa, reforçada por uma série de reforços, podendo esses serem do tipo enrijecedores, nervuras, vigas, longarinas, dentre outros. Esses elementos são dispostos longitudinalmente e, por vezes, nas direções ortogonais [1].

Quando aplicada uma força de pequena amplitude ao longo do comprimento da borda transversal da placa, as deformações serão contidas no próprio plano médio da mesma, resultando em um deslocamento lateral w nulo. Porém, o aumento da magnitude dessa carga altera o modo 
de deformação da placa ocasionando a flambagem. Sendo assim, esse fenômeno acontece quando a peça sofre flexão transversal devido à compressão axial. Devido sua instabilidade ser de caráter elástico, a peça pode perder sua estabilidade sem que o material tenha atingido a tensão de escoamento $\sigma_{y}$. O fenômeno de flambagem acontece em torno do eixo de menor momento de inércia da seção transversal do elemento. Além disso, para cada caso, existe um determinado valor de carga que começa a causar deslocamentos transversais ao plano médio da placa, conhecida como carga crítica. A carga crítica depende, principalmente da esbeltez do membro a ser analisado $[2,3]$.

A utilização de enrijecedores é uma prática bastante comum, porém, esse processo carece de equações e soluções analíticas que realizem seus dimensionamentos. Submetidos à flambagem, a complexidade de dimensionamento é explicada através da dificuldade da análise do elemento esbelto sob carregamentos axiais, bem como, da configuração flambada dos mesmos, uma vez que envolve uma grande quantidade de parâmetros geométricos [4].

Tendo essa barreira em se obter resultados através de soluções analíticas, a simulação numérica torna-se uma importante ferramenta para a análise do comportamento mecânico desses componentes estruturais. Dentre diferentes metodologias o Método dos Elementos Finitos (MEF), que é amplamente empregado para a análise numérica de estruturas, pode ser adotado para o estudo da flambagem de placas com enrijecedores. O MEF baseia-se no método dos deslocamentos e na discretização de uma estrutura em sub-estruturas. Cada uma dessas subestruturas designa-se por elemento finito e tem comportamento conhecido, sendo o comportamento do todo considerado como a soma do comportamento das partes [5].

Sabe-se, entretanto, que modelos computacionais precisam ser verificados e/ou validados. A verificação consiste em comparar os resultados do modelo computacional com soluções analíticas ou com resultados obtidos em outros modelos numéricos. Já a validação é um processo onde os resultados gerados pelo modelo computacional são comparados aos obtidos através de ensaios experimentais.

Diante do exposto, o presente trabalho tem como objetivo realizar a verificação de um modelo computacional para a análise da tensão crítica de flambagem em placas com enrijecedores retangulares. Um modelo computacional para placas sem enrijecedores foi também verificado. As estruturas serão simuladas numericamente através do MEF no software ANSYS, usando um elemento finito do tipo casca (SHELL93).

\section{FLAMBAGEM DE PLACAS}

Em diversas situações de projetos, placas finas são submetidas a cargas de compressão uniaxial, de forma que a grande esbeltez desses componentes faz com que eles sejam suscetíveis a instabilidades como flambagem Para uma placa sem enrijecedores, simplesmente apoiada nos quatro lados e submetida a um carregamento de compressão uniaxial, sua tensão crítica de flambagem $\left(\sigma_{c r}\right)$ pode ser determinada analiticamente por $[6,7,8,9]$ :

$$
\sigma_{c r}=K_{q} \frac{\pi^{2} E}{12\left(1-v^{2}\right)}\left(\frac{t}{b}\right)^{2}
$$

Sendo $E$ o módulo de elasticidade do material, $v$ o coeficiente de Poisson, $t$ a espessura da placa, $b$ o comprimento da borda transversal carregada e $K_{q}$ o coeficiente de flambagem da placa, dado por:

$$
K_{q}=\left(\frac{m}{n} \frac{b}{a}+\frac{n}{m} \frac{a}{b}\right)^{2}, m=1,23, \ldots
$$

onde $a$ é o comprimento da borda longitudinal descarregada da placa; $m$ o número de semi-ondas durante a flambagem na direção de $\sigma_{c r}$, e $n$ na direção transversal à $\sigma_{c r}$, considerado igual a 1 , ou seja, formando apenas uma semi-onda transversal. Para os valores de $m$, tem-se valores de $K_{q}$ em função da relação entre os lados da placa $(a / b)$. Considerando uma placa simplesmente apoiada, para qualquer valor de $m$, o valor de $K_{q}$ tende para um valor mínimo, sendo neste caso $K_{q}=4$ $[10,11]$. 
As cargas coplanares relativamente baixas que uma placa pode suportar sem sofrer flambagem podem ser consideravelmente aumentadas pela adição de enrijecedores. Porém, a inclusão de enrijecedores, acarretam dificuldades encontradas na análise estrutural da placa, devido ao aumento do número de variáveis, além disso, os modos de flambagem são normalmente mais complexos [12]. Consequentemente, na prática da engenharia fórmulas mais simplificadas ou gráficos são utilizados para o projeto de tais placas.

Enquanto enrijecedores longitudinais dispostos paralelamente às cargas coplanares suportam parte da força aplicada, enrijecedores transversais são usados meramente para subdividir a placa em unidade menores, uma vez que, a carga suportada por eles é relativamente pequena. Combinações de enrijecedores paralelos e transversais resultam em placas com enrijecedores ortogonais, chamadas de placas ortotrópicas.

É possível obter a solução analítica para a tensão crítica em uma placa na presença de enrijecedores. Em seus estudos, os mesmos apresentam equações, que variam mediante as condições de contorno da placa, carregamento e número de reforços, as quais permitem a solução analítica de painéis enrijecidos $[13,14]$. Na presença de enrijecedores, o fator $K_{q}$ dependerá das propriedades geométricas tanto da placa, quanto do enrijecedor. Além disso, para determiná-lo, faz-se necessário o cálculo dos seguintes parâmetros:

$$
\begin{aligned}
& \beta=\frac{a}{b} \\
& \delta=\frac{t_{s} h_{s}}{b t} \\
& \gamma=\frac{E I}{b D}
\end{aligned}
$$

onde $a, b$ e $t$ são, respectivamente, o comprimento, a largura e espessura do painel, enquanto $t_{s}, h_{s}$ e $I$ são, respectivamente, a espessura, altura da alma do enrijecedor e seu momento de inércia.

Tendo os parâmetros da placa, o fator $K_{q}$ pode ser calculado a partir da seguinte equação:

$$
\left(K_{q} \beta^{2}\right)^{2}(1+4 \delta)-\left(K_{q} \beta^{2}\right)[(1+2 \delta)(c+d)-8 \gamma \delta]+c d-4 \gamma \delta=0
$$

sendo os parâmetros $c$ e $d$ utilizados como fator de redução de equação e podem ser expressos por:

$$
\begin{aligned}
& c=\left(1+\beta^{2}\right)^{2}+2 \gamma \\
& d=\left(1+9 \beta^{2}\right)^{2}+2 \gamma
\end{aligned}
$$

Para uma placa com um enrijecedor longitudinal a tensão crítica de flambagem é dada pela Eq. (1), com o valor do coeficiente de flambagem $K_{q}$ corrigido dado pela Eq. (6).

Além disso, [13] apresenta a solução analítica para a tensão crítica de uma placa sob compressão com a presença de um enrijecedor transversal, cujo valor é dado por:

$$
\sigma_{c r}=\frac{\pi^{2} D\left(1+\beta^{2}\right)^{2}+2 \gamma \beta^{2}}{t b^{2} \beta^{2}}
$$

A Eq. (9) mostra como a tensão crítica é afetada pela presença do enrijecedor se a placa flamba em uma meia onda.

Por fim, Szilard (2004) [12] apud Wittrick (1952) [15] apresentou uma solução aproximada para uma placa na presença de enrijecedores longitudinais e transversais baseando-se na Teoria de Placas Ortotrópicas.

Em seu trabalho, Wittrick (1952) [15] derivou cargas críticas de placas retangulares reforçadas com diferentes condições de contorno utilizando a idealização do problema de placas ortotrópicas. Se os reforços longitudinais são flexíveis o suficiente para permitir a flambagem global da placa, consequentemente, a tensão crítica pode ser estimada por: 


$$
\left(\sigma_{c r}\right)=\frac{4 \pi^{2} E}{12\left(1-v^{2}\right)}\left(\frac{h}{b}\right)^{2}\left\{1+\left[1+12\left(1-v^{2}\right) \frac{I_{x}}{c_{1} t^{3}}\right]^{1 / 2}\right\}
$$

assumindo-se que os reforços longitudinais se encontram na direção $x$, onde que $I_{x}$ é o momento de inércia das nervuras considerando como referência a superfície média da placa, e $c_{l}$ os espaçamentos das nervuras nas direção $x$.

\section{MODELAGEM COMPUTACIONAL}

Nos últimos anos, métodos computacionais têm sido amplamente empregados em análises estruturais. Quando se analisa problemas de painéis sob esforços mecânicos, suas soluções são praticamente impossíveis de se resolver quando se tenta por meio da aplicação das equações diferenciais da teoria da elasticidade. Para a análise desses problemas, se procura outros métodos de solução [16]. Um destes métodos tem sido designado no Método dos Elementos Finitos (MEF), o qual resulta soluções aproximadas para tais problemas.

No MEF os elementos são conectados entre si, na presença de nós localizados em suas extremidades, fazendo com que seu comportamento da malha formada pelo conjunto de elementos seja análogo ao contínuo original [17]. A determinação dos deslocamentos nodais se dá por um conjunto de equações algébricas de equilíbrio, formando um sistema de equações equivalente à malha utilizada. A solução do sistema pode ser obtida aplicando-se condições de contorno externas do problema.

A teoria sobre o MEF é bastante difundida, podendo ser encontrada facilmente em referências como [18], [19] e [20].

O software ANSYS, que é baseado no MEF, foi empregado no desenvolvimento dessa pesquisa. Como o estudo é relacionado a placas finas, optou-se pelo uso do elemento SHELL93 (Fig. 1), que é um elemento do tipo casca estrutural 2D.

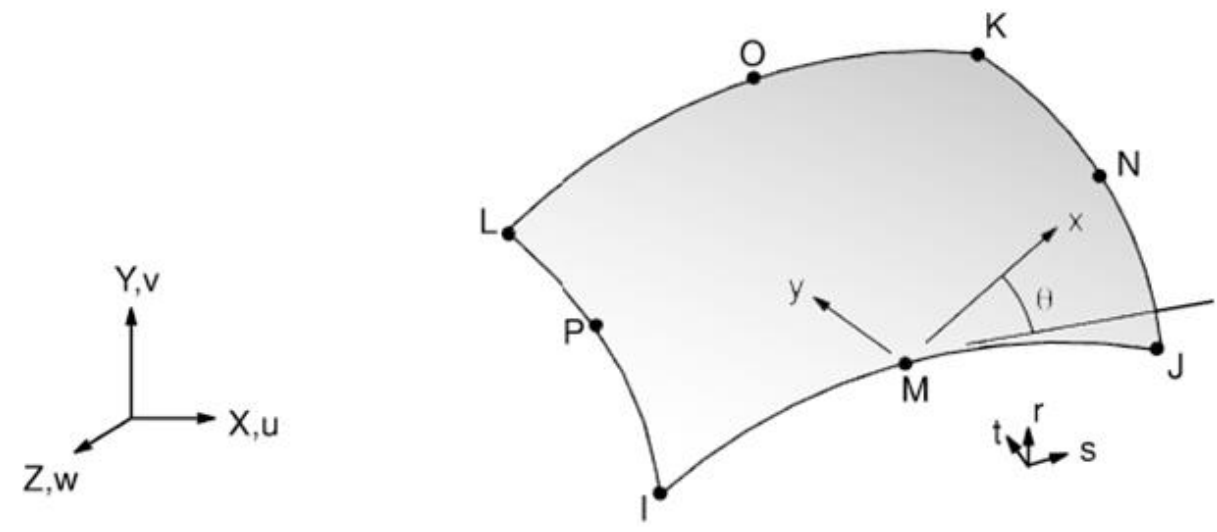

Figura 1: Elemento finito SHELL93 [21]

O elemento SHELL93 é um elemento finito do tipo casca de caráter isoparamétrico. Cada um de seus oito nós possui seis graus de liberdade, sendo três translações nas direções $x, y$ e $z$, além de três rotações também em torno desses eixos [22]. Caracterizado como um elemento finito 2D, é considerado de caráter tridimensional devido à sua não restrição ao plano $x$-y, podendo ser empregado em qualquer posição do espaço tridimensional, além de ser deformável na direção fora de seu plano. Os elementos finitos tipo casca são usados para modelar componentes estruturais do tipo placa que possuem espessura pequena se comparada às suas outras dimensões. Podem ser carregados em seu plano (carregamentos de membrana) bem como fora de seu plano por momentos fletores e torsores. Além disso, o elemento finito SHELL93 pode considerar grandes deslocamentos e plasticidade [22, 23].

A necessidade de uma grande acuracidade no resultado e a qualidade da malha a ser trabalhada são fatores preponderantes que possibilitam a diferenciação dos valores aceitáveis para os desprezíveis. Para a verificação da malha a ser adotada considerou-se uma placa com 
enrijecedores, onde se adotou elementos regulares do tamanho de $20 \mathrm{~mm}$, tamanho esse definido a partir de um teste de convergência de malha. $\mathrm{O}$ esquema de malha adotado é apresentado na Fig. 2.

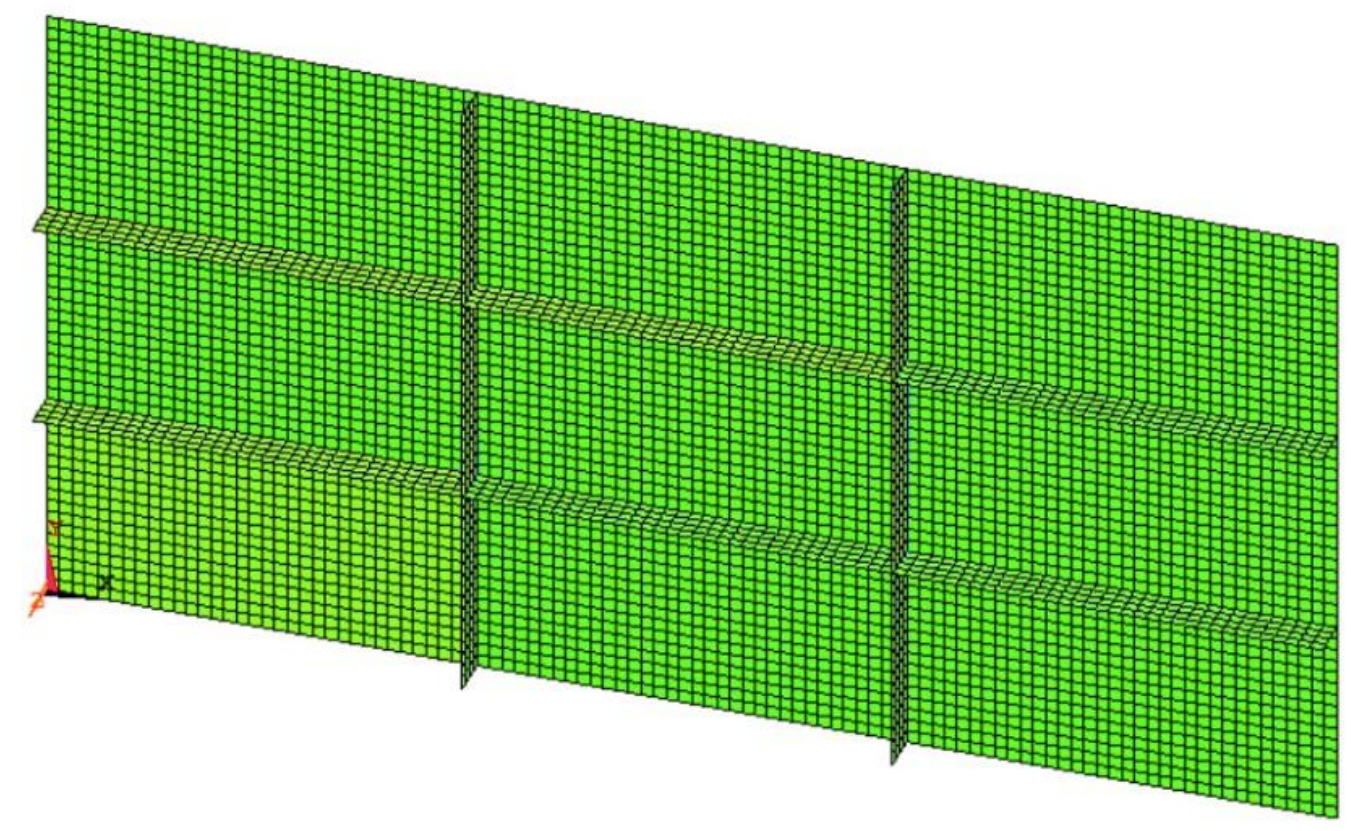

Figura 2: Malha do tipo quadricular para a placa retangular enrijecida

Para a análise numérica de flambagem foi adotado o método elástico de autovalores. A solução de equações algébricas homogêneas foi utilizada para analisar as equações de equilíbrio por elementos finitos para esse tipo de análise [24]. O autovalor mais baixo corresponde à carga crítica de flambagem e o autovetor a ele associado representa o primeiro modo de flambagem.

Para essa formulação,são considerados tanto os termos lineares como os não-lineares. Assim, tem-se a matriz de rigidez total $[K]$, que é obtida pela soma da matriz de rigidez convencional para pequenas deformações, $\left[K_{E}\right]$, com a matriz de rigidez geométrica $\left[K_{G}\right]$. A matriz $\left[K_{G}\right]$ além da geometria, depende também do esforço normal de compressão existente no início do carregamento, $\left\{P_{0}\right\}$. Sendo assim, a placa irá possuir um deslocamento total $\{U\}$ associado a um nível de carga $\left\{P_{0}\right\}$ que é dado por [25]:

$$
\{U\}=\left[\left[K_{E}\right]+\lambda\left[K_{G}\right]\right]^{-1} \lambda\left\{P_{0}\right\}
$$

onde $\lambda$ é um escalar.

$\mathrm{Na}$ análise de flambagem, a placa tem um grande crescimento nos deslocamentos sem crescimento da carga. Matematicamente é possível determinar a matriz inversa de forma que, os deslocamentos $\{U\}$ tendem a infinito quando:

$$
\operatorname{det}\left[\left[K_{E}\right]+\lambda\left[K_{G}\right]\right]=0
$$

A Eq. (12) representa um problema de autovalores, que ao ser resolvido gera o menor autovalor, $\lambda_{1}$, que corresponde à carga crítica $\left\{P_{c r}\right\}=\lambda_{1}\left\{P_{0}\right\}$ ocorrendo flambagem. Além disso, o vetor de deslocamento associado $\{U\}$ define o modo de flambagem. O problema de autovalores é resolvido usando o método numérico de Lanczos [26].

\section{RESULTADO E DISCUSSÕES}

Atualmente a análise da flambagem de placas enrijecidas é robustamente feita através da utilização de métodos numéricos, tal como a análise de Elementos Finitos [27].

A seguir são apresentadas umas comparações entre soluções numéricas e analíticas propostas na literatura. Antes disso é apresentada também a comparação entre as soluções numérica e analítica para a flambagem elástica de uma placa sem enrijecedores. 
Para a verificação do modelo computacional utilizou-se uma placa maciça simplesmente apoiada de espessura constante sob flambagem. De acordo com a Fig. 3, este painel possui comprimento $a=2000 \mathrm{~mm}$, largura $b=1000 \mathrm{~mm}$ e espessura $t=20 \mathrm{~mm}$.

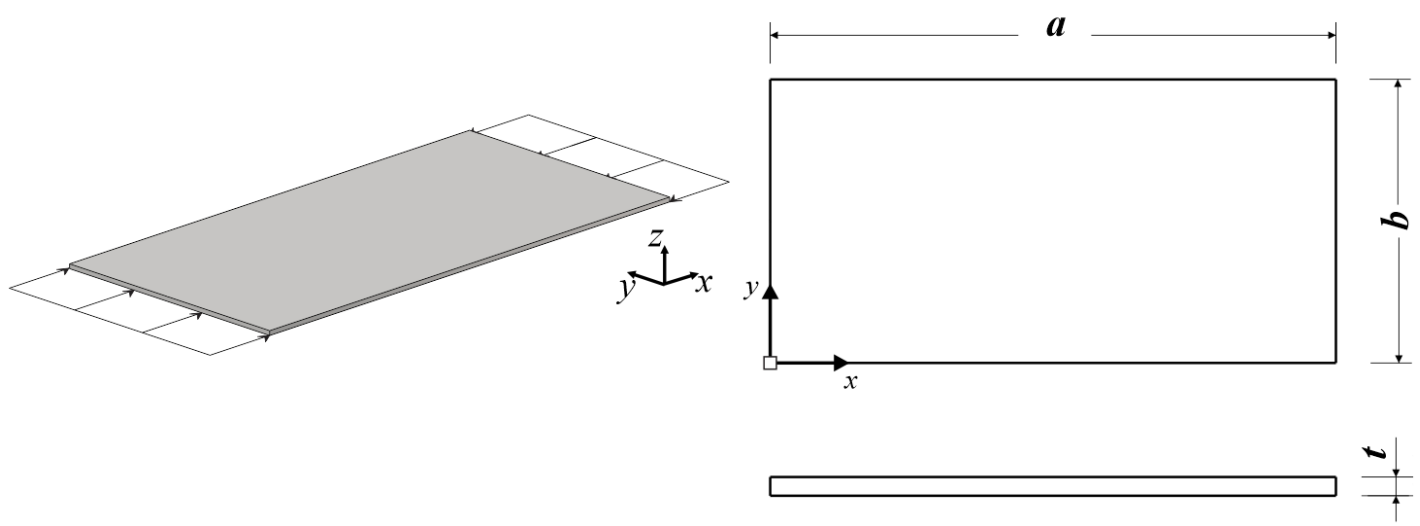

Figura 3: Detalhamento da placa maciça sob compressão uniaxial, em $\mathrm{mm}$

Para todos os casos analisados adotou-se o material Aço AH-36, o qual possui valores de tensão de escoamento, módulo de elasticidade e coeficiente de Poisson, respectivamente, $\sigma_{y}=355$ $\mathrm{MPa}, \quad E=210 \mathrm{GPa}, v=0,3$.

A solução analítica é proposta por [11] e é definida pela Eq. (1). Para a placa proposta, a tensão crítica de flambagem determinada pela solução analítica é $\sigma_{c r}=303,68 \mathrm{MPa}$. Já através da modelagem computacional, foi obtida uma tensão crítica como sendo $\sigma_{c r}=299,00 \mathrm{MPa}$. Portanto, uma diferença de $1,54 \%$ entre o modelo numérico e a solução analítica foi encontrada, verificando o modelo computacional. Na Fig. 4 a configuração flambada para a placa sem enrijecedores é apresentada, onde as cores vermelha e azul indicam, respectivamente, as maiores deflexões no sentido positivo e negativo do eixo normal à placa (eixo $z$ ). Esse código de cores será usado em todas as configurações flambadas apresentadas.

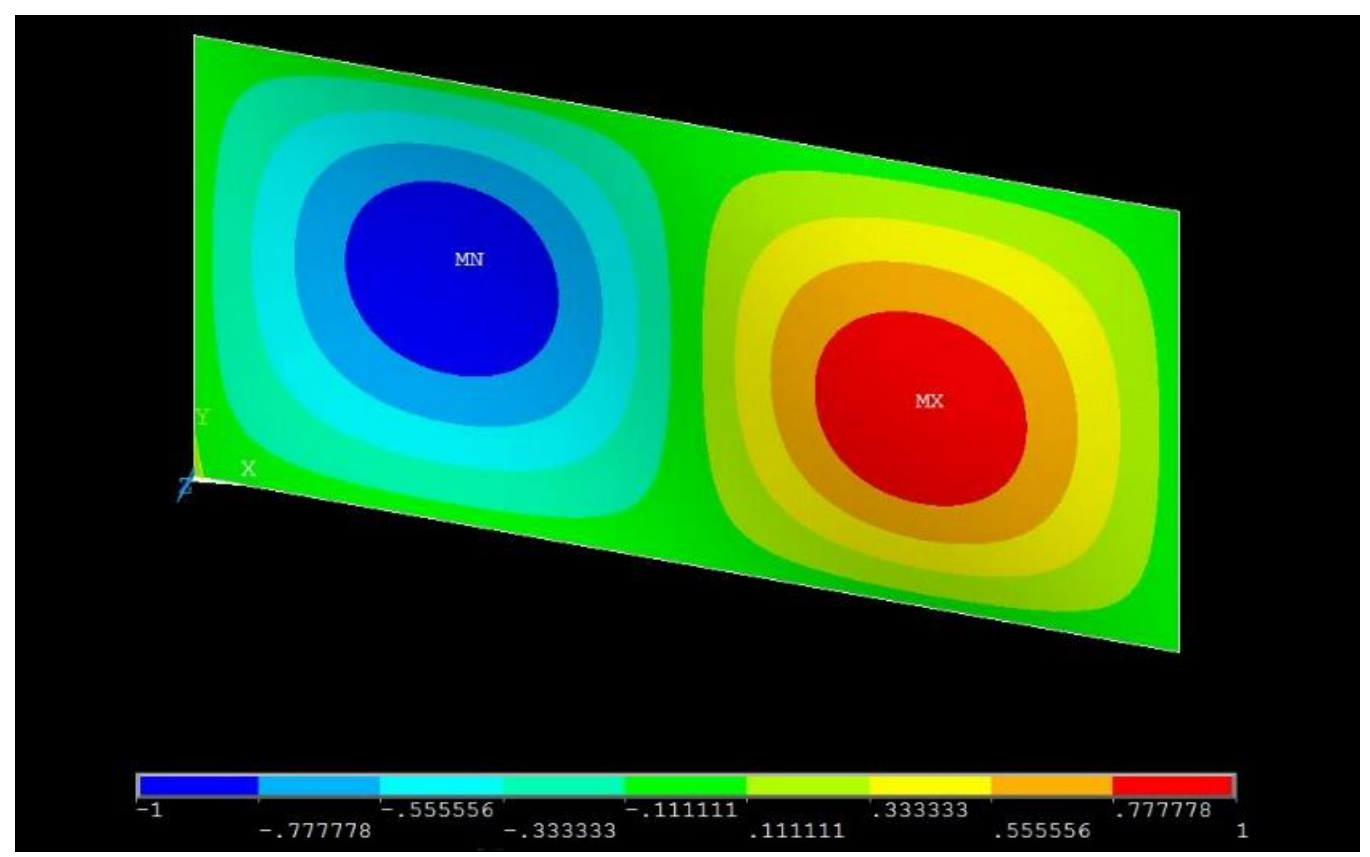

Figura 4: Configuração flambada para a placa maciça

Já primeira análise é feita com um painel retangular fino de espessura constante sob compressão no plano, contendo em seu eixo central, um enrijecedor longitudinal proposto por [3]. De acordo com a Fig. 5, este painel simplesmente apoiado possui comprimento $a=1000 \mathrm{~mm}$, largura $\quad b=500 \mathrm{~mm}$ e espessura $t=9 \mathrm{~mm}$. O enrijecedor presente possui altura $h_{s}=$ $50 \mathrm{~mm}$, espessura $\quad t_{s}=9 \mathrm{~mm}$, e comprimento $l_{s}$ igual ao comprimento da placa. 

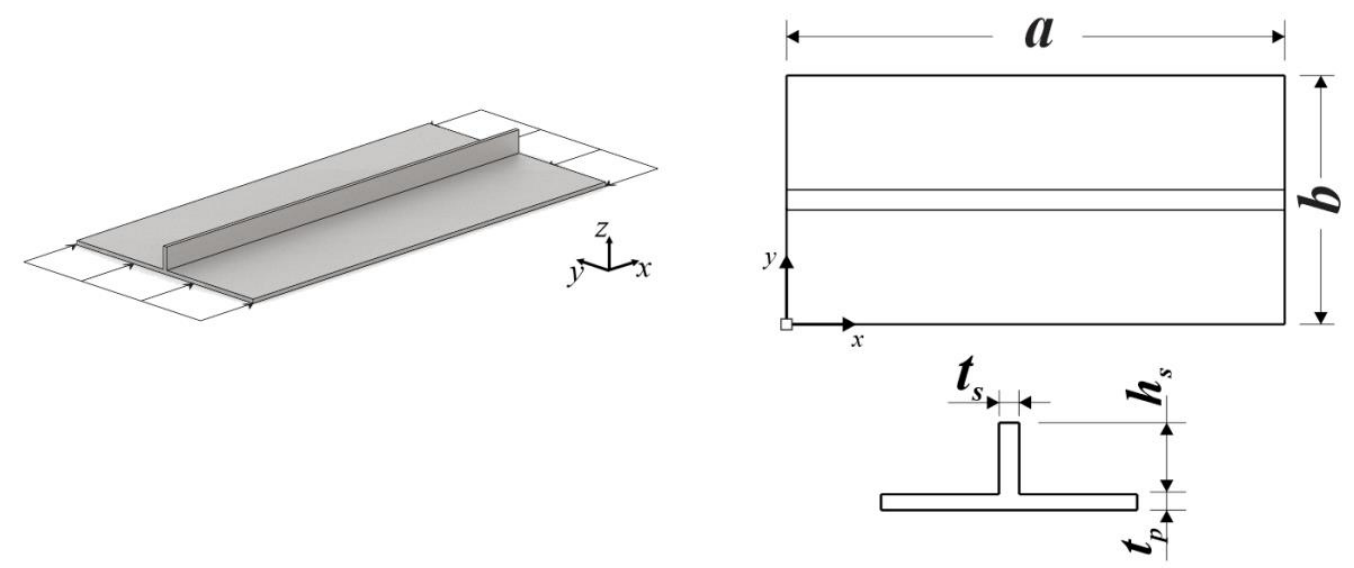

Figura 5: Detalhamento da placa com um enrijecedor longitudinal para flambagem elástica, em mm

Nesse estudo realizou-se uma verificação linear sobre flambagem, cuja solução analítica é obtida pelas Eqs. (1) e (6). Para a placa proposta, a tensão crítica de flambagem determinada pela solução analítica é $\sigma_{c r}=606,12 \mathrm{MPa}$. Através da modelagem computacional, tem-se a tensão crítica como sendo $\sigma_{c r}=624,36 \mathrm{MPa}$, cujo modo de flambagem elástica pode ser verificado na Fig. 6. Para o estudo em questão foi-se obtido uma diferença de 3,01\% entre os valores numérico e analítico.

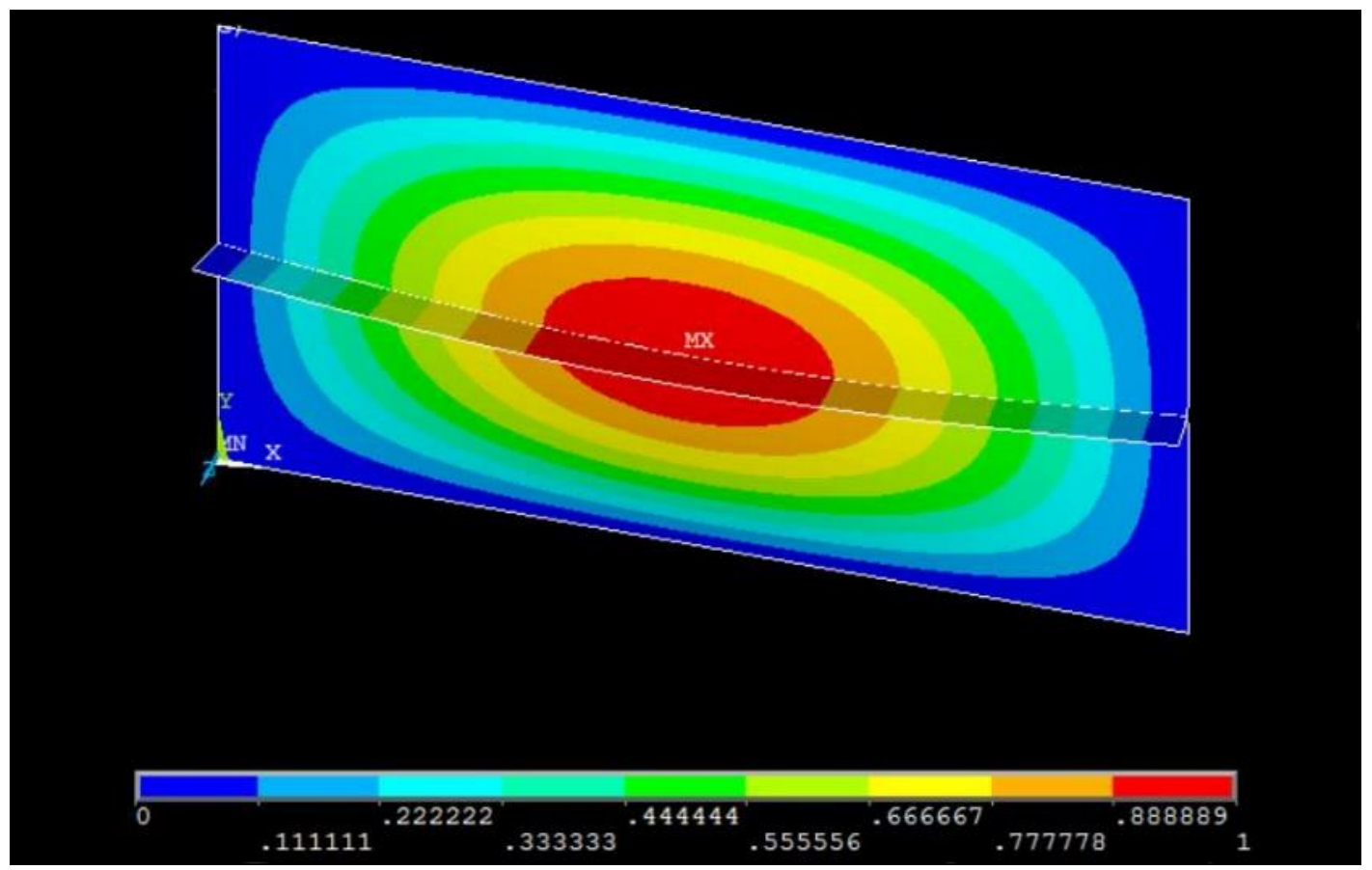

Figura 6: Configuração deformada para a placa com um enrijecedor longitudinal

Após, na análise de placas com um enrijecedor transversal utilizou-se um painel quadrado, com espessura constante, sob compressão no plano, possuindo um enrijecedor central posicionado paralelamente à aplicação da carga. De acordo com a Fig. 7, este painel simplesmente apoiado possui comprimento $a=1000 \mathrm{~mm}$, largura $b=1000 \mathrm{~mm}$ e espessura $t=10 \mathrm{~mm}$. O enrijecedor presente possui altura $h_{s}=100 \mathrm{~mm}$ e espessura $t_{s}=10 \mathrm{~mm}$, além disso, seu comprimento $l_{s}$ é equivalente à largura $a$ da placa.

Nessa análise de flambagem elástica na placa, a solução analítica é proposta por Szilard (2004) [12], e é dada pela Eq. (9). Para a placa proposta, a tensão crítica de flambagem determinada pela 
solução analítica é $\sigma_{c r}=121,09 \mathrm{MPa}$. Na modelagem computacional, obteve-se a tensão crítica como sendo $\sigma_{c r}=121,32 \mathrm{MPa}$, cujo modo de flambagem elástica pode ser visto na Fig. 8 .

Analisando esse estudo foi possível observar uma diferença de $0,18 \%$, entre os valores numérico e analítico.
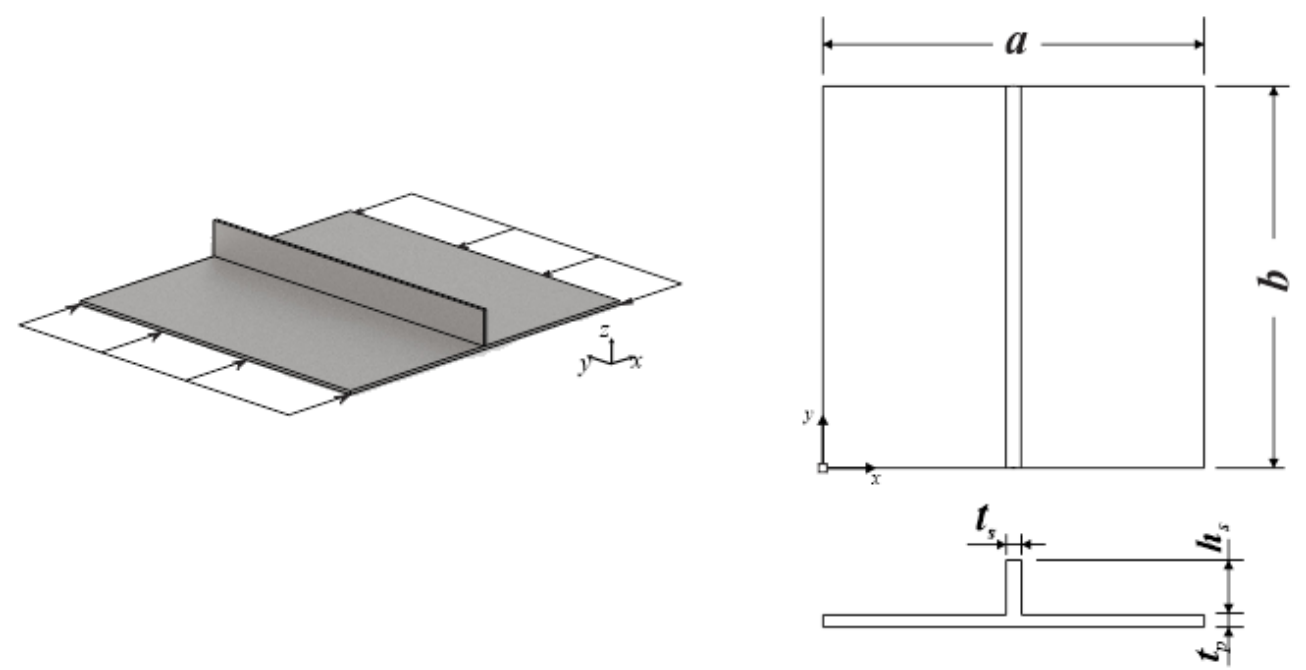

Figura 7: Detalhamento da placa com um enrijecedor transversal para flambagem elástica, em mm

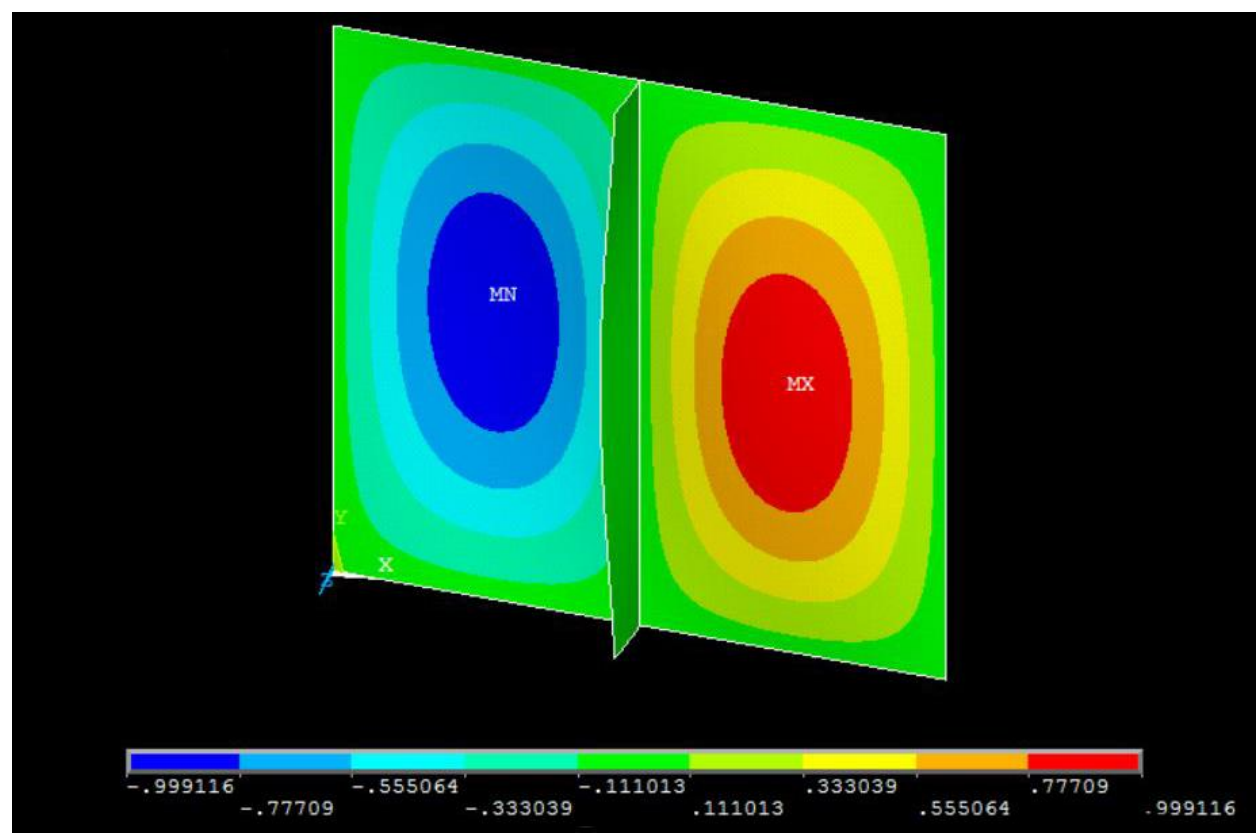

Figura 8: Situação deformada para a placa com um enrijecedor transversal

Por fim, analisou-se a situação em que uma fina placa retangular com espessura constante, sob compressão no plano, possui enrijecedores dispostos longitudinalmente e transversalmente, contendo quatro em ambas as direções. De acordo com a Fig. 9, este painel simplesmente apoiado possui comprimento $a=2000 \mathrm{~mm}$, largura $b=1000 \mathrm{~mm}$ e espessura $t=10 \mathrm{~mm}$. Os enrijecedores possuem altura $h_{s}=50 \mathrm{~mm}$ e espessura $t_{s}=10 \mathrm{~mm}$. Os mesmos foram dispostos simetricamente em relação ao eixo central do plano horizontal da placa, de forma que para o eixo longitudinal e transversal, os espaçamentos entre os enrijecedores foram de $c_{x}=200 \mathrm{~mm}$ e $c_{y}=400 \mathrm{~mm}$.

Para a placa da Fig. 9, considerando a Eq. (10), a tensão crítica de flambagem determinada pela solução analítica é $\sigma_{c r}=351,36 \mathrm{MPa}$. Já através da simulação numérica, obteve-se uma tensão crítica de $\sigma_{c r}=349,83 \mathrm{MPa}$. Portanto, uma diferença de 0,43\% foi encontrada ao comparar a 
solução numérica com a analítica. Na Fig. 10 é apresentada a configuração flambada para a placa da Fig. 9.
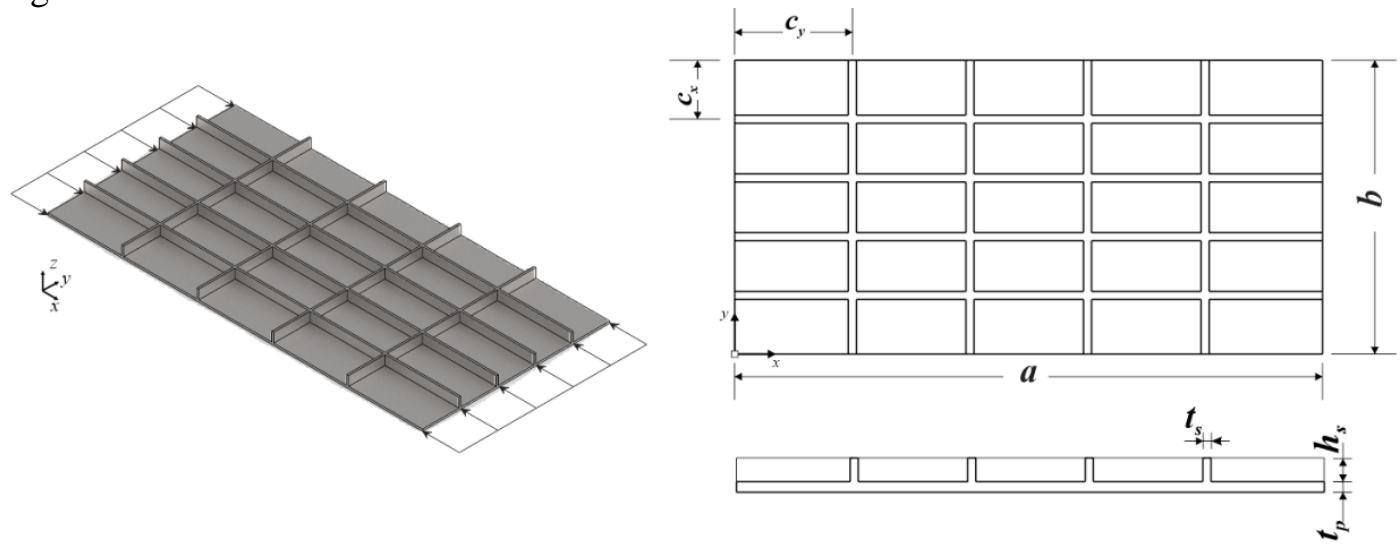

Figura 9: Detalhamento da placa ortotrópica para flambagem elástica, em mm

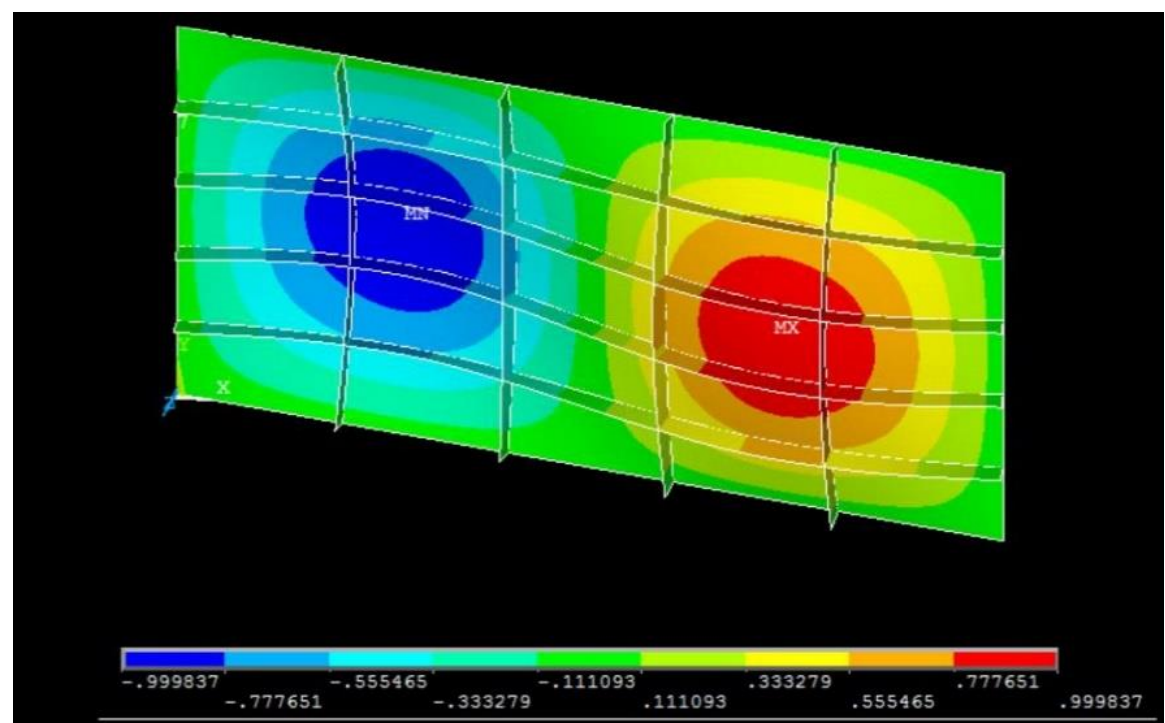

Figura 10: Situação deformada para a placa ortotrópica

\section{CONCLUSÕES}

Na Engenharia Estrutural têm-se situações cujas análises por métodos analíticos se tornam inviáveis. Seja pela dificuldade ou tempo necessário ao desenvolvimento de equações matemáticas, ou até mesmo em situações em que se tenha a ausência de uma análise analítica para o problema exposto. Sendo assim, através da escolha de um correto modelo matemático, bem como de uma técnica computacional ideal, é possível obter através da modelagem computacional resultados de grande credibilidade ao comportamento mecânico proposto.

Empregando o software ANSYS, o qual utiliza do Método dos Elementos Finitos, foi possível simular numericamente o comportamento mecânico de placas sem e com enrijecedores submetidas à flambagem elástica. Os modelos computacionais, tanto para a placa sem enrijecedores, como para placa com enrijecedores, foram devidamente comparados com as respectivas soluções analíticas.

Através dos resultados obtidos, pode-se concluir que a utilização do modelo proposto para a análise numérica computacional das placas enrijecidas foi satisfatória, uma vez que, para os casos estudados, foram encontradas diferenças entre os modelos numéricos e analíticos de 1,54\%, 3,1\%, $0,18 \%$ e $0,43 \%$, respectivamente, para a placa inicialmente sem enrijecedores, placa com um enrijecedor longitudinal, placa com um transversal e placa ortotrópica enrijecida longitudinalmente e transversalmente, respectivamente. Sendo assim, é possível concluir que os 
modelos computacionais foram devidamente verificados e podem ser empregados para a análise numérica desses componentes estruturais.

Para trabalhos futuros, tem-se como proposta a análise do comportamento elasto-plástico dos modelos propostos, bem como a análise de estudos experimentais, comparando os resultados utilizando a modelagem computacional, bem como a aplicação desses modelos computacionais considerando diferentes tipos de enrijecedores e em estudos de otimização geométrica

\section{AGRADECIMENTOS}

Os autores agradecem à CAPES e ao $\mathrm{CNPq}$ pelas bolsas concedidas que permitiram o desenvolvimento dessa pesquisa.

\section{REFERÊNCIAS BIBLIOGRÁFICAS}

1. Bedair O. Analysis and Limit State Design of stiffened plates and shells: A world view. Applied Mechanics Reviews. 2009; 62, doi:10.1115/1.3077137.

2. Abed FH, Megahed M, Al-Rahmani A. On the improvement of buckling of pretwisted universal steel columns. Structures. 2016; 5:152-160, doi: dx.doi.org/10.1016/j.istruc.2015.10.012.

3. Pfeil W, Pfeil M. Estruturas de Aço: Dimensionamento prático. Rio de Janeiro: LTC. $8^{\mathrm{a}}$ ed., 2012.

4. Rackow PB. Análise numérica não-linear de painéis com enrijecedores sob flambagem [dissertação]. Rio Grande (RS): Universidade Federal de Rio Grande; 2015. 115 p.

5. Azevedo AFM. Método dos elementos finitos. Porto: Faculdade de Engenharia da Universidade do Porto, 2003. $1^{\text {a }}$ ed. 248 p.

6. Åkesson B. Plate buckling in bridges and other structure, Londres: Taylor \& Francis, 2007. 164 p.

7. El-Sawy KM, Nazmy AS. Effect of aspect ratio on the elastic buckling of uniaxially loaded plates with eccentric holes. Thin-Walled Structures, 2001; 39:983-998,doi:dx.doi.org/10.1016/S02638231(01)00040-4.

8. Wang CM, Wang CY, Reddy JN. Exact solutions for buckling of structural members. Flórida: CRC Press 2005. $207 \mathrm{p}$.

9. Real MV, Isoldi, LA. Finite element buckling analysis of uniaxially loaded plates with holes. Porcedente de Southern Conference on Computational Modeling, 2010, Rio Grande (RS). p. 69-73.

10. Trahair NS, Bradford MA, Nethercot DA, Gardner L. The behavior and design of steel structures, Londres: Taylor \& Francis, 2007. $4^{\mathrm{a}}$ ed, $490 \mathrm{p}$.

11. Salmon CG, Johnson, JE, Malhas FA. Steel structures: Design and behaviour: Emphasizing load and resistance factor design. Prentice Hall, 2009. 866p

12. Szilard R. Theories and applications of plate analysis: Classical numerical and engineering methods. New Jersey: John Wiley \& Sons, Inc., 2004. 1024 p.

13. Timoshenko, SP; Gere, JM. Theory of elastic stability. New York: McGraw - Hill, 1985. 541 p.

14. Bares R. Tablas para el cálculo de placas y vigas pared. Barcelona: Gustavo Gili, S.A., 1981. 538 p.

15. Wittrick WH. Correlation between some stability problems for orthotropic and isotropic plates under biaxial and uniaxial stress, The Aeronautical Quarterly. 1952, 4: 83-92.

16. Manrique LJC. Colapso de painéis planos enrijecidos. [dissertação]. Rio de Janeiro (RJ): Universidade Federal do Rio de Janeiro; 1989. 126 p.

17. Rao SS. The finite element method in engineering. Miami: Elsevier Science \& Technology Books, 2004. $726 \mathrm{p}$.

18. Zienkiewicz OC. The finite element method in engineering science. London: McGraw-Hill, 1971. 521 p.

19. Gallagher RH. Finite element analysis: Fundamentals. Englewood Cliffs: Prentice-Hall, 1975. 416 p.

20. Bathe KJ. Finite element procedures. Watertown: Prentice- Hall, 2006. 1046 p.

21. Kohnke P. ANSYS theory reference - Release 5.6. ANSYS, Inc., 1999. $11^{\mathrm{a}} \mathrm{Ed}$.

22. Dufour P. Picking an element type for structural analysis. Belcan Engineering Group, Inc., 2003.p. 5.

23. Ross, CTF. Pressure vessels: External pressure technology. Padstow: Woodhead Publishing, $2^{\mathrm{a}}$ Ed., 2011. $488 \mathrm{p}$.

24. Madenci E, Guven I. The finite element method and applications in engineering using ANSYS®. Springer, 2006. 657p.

25. Przemieniecki JS. Finite element structural analysis: New concepts. Reston: AIAA (American Institute of Aeronautics \& Astronautics), 2009. 138 p.

26. ANSYS User's Manual (version 10.0), 2005. Houston. Swanson Analysis System Inc. 
27.Ziemian RD. Guide to stability design criteria for metal structures. John Wiley Sons. Hoboken. 2010. $1078 \mathrm{p}$. 